\title{
Expanded Endoscopic Endonasal Approach and Adjuvant Radiotherapy for the Treatment of Locally Advanced Sinonasal and Skull Base Adenoid Cystic Carcinoma: Case Report and Literature Review
} \author{
${ }^{1}$ Department of Radiation Oncology, Instituto Nacional de Cancerología, Colombia \\ ${ }^{2}$ Department of Head and Neck Surgery, Instituto Nacional de Cancerología, Colombia \\ ${ }^{3}$ Chief of Radiation Oncology department, Instituto Nacional de Cancerología, Colombia \\ ${ }^{4}$ Neurosurgeon, Endoscopic skull base surgery group, Instituto Nacional de Cancerología, Colombia \\ ${ }^{5}$ General Practitioner, University of Antioquia, Colombia \\ ${ }^{6}$ Otolaryngologist, skull base endoscopic surgery group, National Cancer Institute, Colombia
}

Alexandra Vallejo ${ }^{1}$, Judith Vásconez Escobar ${ }^{2 *}$, José Esguerra ${ }^{3}$, Nicolás Gill ${ }^{4}$ Katherine Vallejo5 and Javier Ospina ${ }^{6}$

Submission: January 07, 2020; Published: January 25, 2021

*Corresponding author: Judith Vásconez Escobar, Department of Head and Neck Surgery, Instituto Nacional de Cancerología, Bogotá D C, Colombia

Abstract

Introduction: Adenoid Cystic Carcinoma of the sinonasal and skull base region is a challenging tumor to treat due to its late onset of presentation and frequent compromise of vital neurovascular structures. Surgical treatment followed by conventional irradiation are the optimal treatment options, often requiring extensive open approaches and mutilating interventions due to its localization. Endoscopic endonasal approaches are relatively new procedures that combined with new adjuvant radiation techniques could achieve similar oncological outcomes with significant less morbidity.

Methods: We present the case of a patient with non-metastatic Adenoid Cystic Carcinoma (ACC) locally advanced of the sinonasal and skull base region diagnosed and treated at the Instituto Nacional de Cancerología of Bogotá D.C, Colombia between the years 2017 and 2020 who underwent endoscopic endonasal tumor resection and subsequent adjuvant management with Intensity modulated radiotherapy (IMRT).

Results: Endoscopic and imaging control was performed according to the protocol of the surgical group with nasal endoscopies every 3-4 months during the first 2 years and serial images every 6 months. Follow up for 35 months with adequate local control and minimal aesthetic or functional sequelae.

Conclusion: Multidisciplinary management with endoscopic endonasal resection and adjuvant radiotherapy appears to be an effective oncological alternative to open surgical procedures, with less morbid and minor functional sequelae, for patients with locally advanced ACC tumors of the sinonasal region.

Keywords: Adenoid cystic carcinoma; Sinonasal carcinoma; Paranasal sinuses; Head and neck cancers; Transnasal endoscopic surgery; Adjuvant radiation

\section{Introduction}

Adenoid cystic carcinoma (ACC) is a rare tumor that generally affects the salivary glands in the head and neck [1], the most common site are the minor salivary glands followed by the paranasal sinuses [2]. ACC can affect all age groups but is most common between 50 and 60 years [3]. Adenoid cystic carcinoma of the head and neck is difficult to control, due to its high propensity for recurrence and distant metastasis. These tumors show high propensity of dissemination to the skull base and cranial nerves, which is found in up to $50 \%$ of patients displaying changes such as bone lysis and perivascular or perineural spread through the skull foramens $[3,4]$. Unfortunately for these patients, this extensive compromise is often found when the first symptoms appear $[5,6]$. 
These tumors are usually diagnosed in a locally advanced stage, as the symptoms can be mistaken as chronic rhinosinusitis or inflammatory changes on CT scan images [7].

Historically, the main treatment for adenoid cystic carcinoma has been surgical oncological resection of the primary tumor followed by adjuvant radiation therapy (RT) [8]. However, in cases of locally advanced tumors with skull base involvement, radical resection with negative margins is often impossible to achieve, which increases the risk for recurrence of the disease. Different studies have shown that treatment with adjuvant RT with curative intention is a therapeutic option that reduces the probability of local recurrence [9], with an improvement in local control of 30 to $80 \%$. This is indicated in patients with T3-T4 tumors, incomplete resection or with close margins (1-5mm), bone involvement, perineural invasion and histological factors for high degree of disease recurrence. The most modern irradiation techniques such as modulated intensity allows to achieve greater conformation of the dose providing optimal therapy with less toxicity. Other randomized studies have shown contradictory results, without a clear benefit of adjuvant therapy in this type of paranasal sinus tumors [5,8,10-12].

Despite the well stablished role for open oncological surgical resection in sinonasal malignant tumors, these techniques are associated with high morbidity and mortality, since the location within the sinuses and skull base requires extensive and mutilating procedures, which limits the complete resection (R0) of this tumor and increases the facial deformity or complications such as CSF (cerebrospinal fluid) leak. The endoscopic transnasal surgery recently appears as a safe and effective approach in selected patients, that reduces the morbidity of open procedures with similar oncological results with the additional advantage of achieving better aesthetic and functional results [13].

\section{Case Report}

60-year-old woman who presented in January 2017 with decreased visual acuity associated with right diplopia. The MRI study of the paranasal sinuses showed an expansive bony appearance tumor with involvement of the sphenoid sinus, skull base, clival region and extending to the suprasellar prepontine cistern measuring 49x 44x42 mm (Figure 1). Surgical resection was taken in place in March 2017 with an extended endonasal endoscopic procedure (trans pterygoid and trans clival approach), finding a rubbery tumor that involved the posterior and middle wall of the right maxillary sinus, right pterygomaxillary fossa and the medial portion of the infratemporal fossa, sphenoid sinus with extension to the posterior cranial fossa eroding the middle and superior clivus. There was also tumor infiltration in bilateral peri carotid tissue. A wide resection of the medial wall of the cavernous sinuses was achieved, maintaining its integrity.

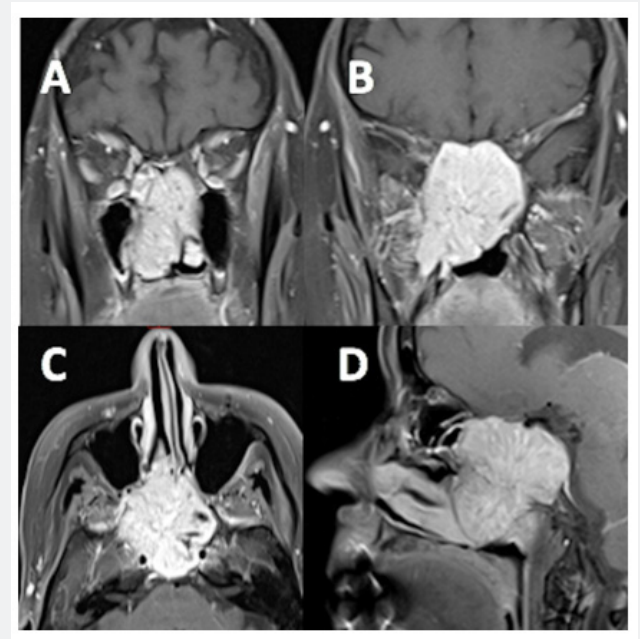

Figure 1: The MRI study of the paranasal sinuses: 2017. An expansive bony appearance tumor with involvement of the sphenoid sinus, skull base, clival region and extending to the suprasellar prepontine cistern measuring 49x44x42mm. A y B: coronal Plane, C: Axial plane y D: Sagital Plane.

Partial involvement of the dura was also observed in the medial region and right paramedian region, requiring a fascia late graft for reconstruction. A median and superior clivectomy was performed and partial resection of the inferior clivus until a macroscopically healthy bone tissue was obtained achieving a macroscopically complete resection at this level (verified with Brainlab $\AA$ neuronavigation system). The Histopathology report indicated adenoid cystic carcinoma, with cribriform pattern, tubular and solid. Histological grade 2, with perineural involvement, immunochemistry reported $\mathrm{CD}_{117}(+) ; \mathrm{P}_{40}(+)$; EMA $(+) ; \mathrm{CK}_{5} / 6(+) ; \mathrm{CK}_{7}(+) ; \mathrm{GATA}_{3}(+)$; $\mathrm{PHH}_{3}$ : up to 4 mitoses/10 high power fields; $\mathrm{ki}_{67}: 25 \%$ which is in relation with the characteristics 


\section{Global Journal of Otolaryngology}

reported in literature (Figure 2). The patient received adjuvant IMRT technique External Radiotherapy with curative intent, doses from 2 Gy to 66 Gy in the surgical bed between June 6, 2017 and July 19, 2017 with adequate tolerance (Figure 3).

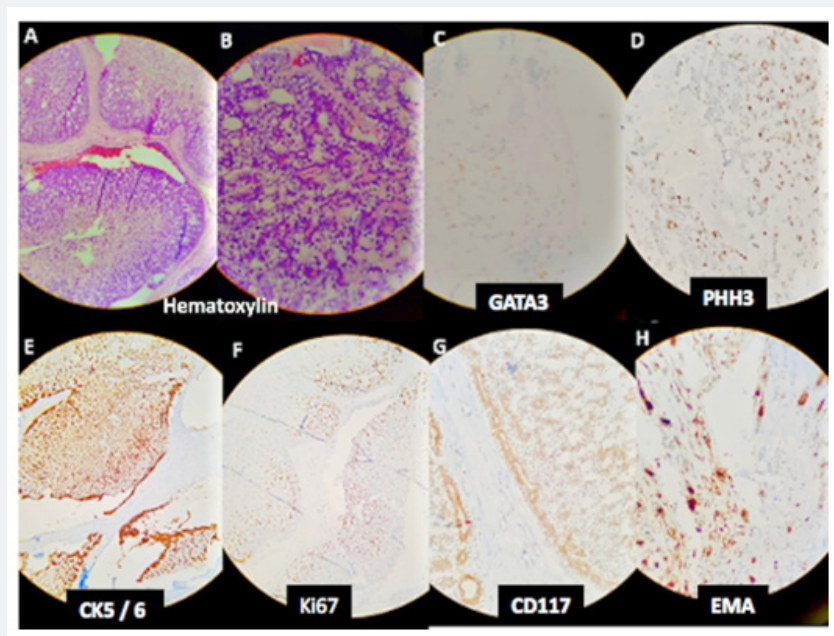

Figure 2: The Histopathology report. Hematoxylin: adenoid cystic carcinoma of cribriform pattern with myoepithelial cells and luminal epithelial cells is identified. The IHQ: Myoepithelial cells are positive for CK5 / 6, epithelial cells positive for EMA, CD117 and GATA3, Ki67 cell proliferation index of $25 \%$ and mitotic activity measured by $\mathrm{PHH} 3$ with 4 mitoses/10 HPF (High power field).

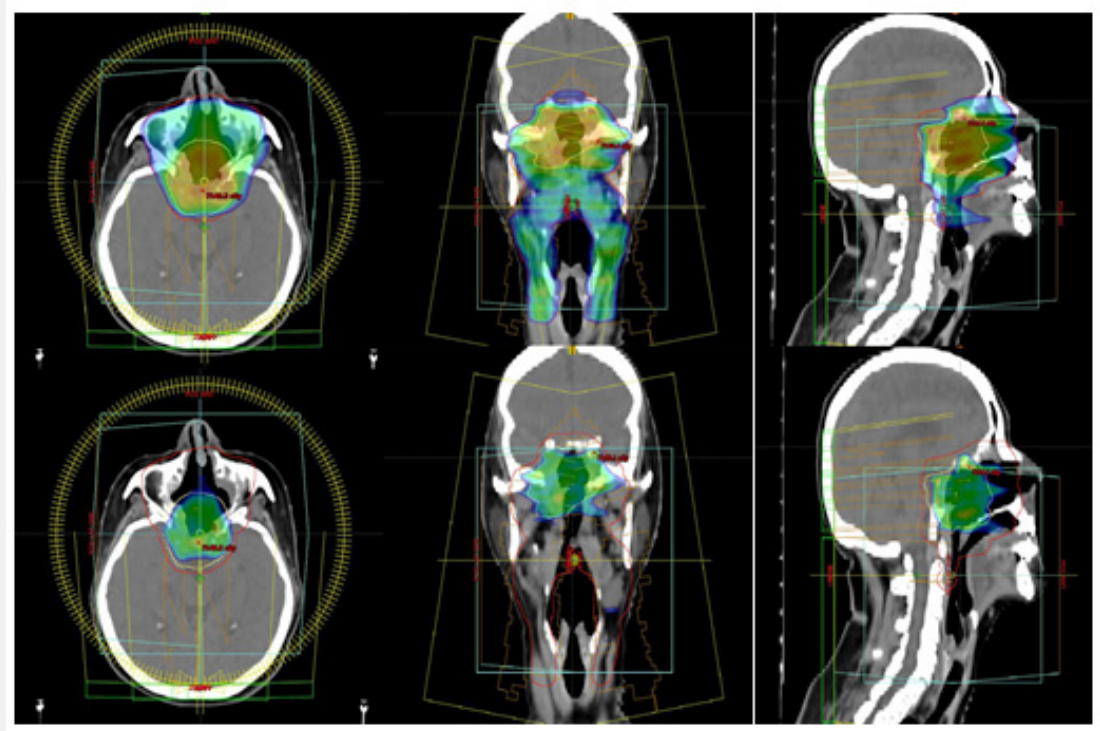

Figure 3: Radiotherapy-VMAT The patient received adjuvant external radiotherapy VMAT with curative intent, doses from 2 Gy to 66 Gy in July, 2017 with adequate tolerance. Two volumes were performed, one elective up to $54 \mathrm{~Gy}$ and a boost to $66 \mathrm{~Gy}$. The yellow and blues lines represent disposition and arc conformation, respectively, in the axial, coronal and sagittal planes. The color wash of the circular-colored area represents the dose delivered to the target volume.

During the follow-up period, endoscopic and imaging control was performed according to the protocol of the surgical group with nasal endoscopies every 3-4 months during the first 2 years and images every 6 months. The latest follow-up images in
January 2020 (35 months after treatment) showed postoperative changes in the endonasal approach with the presence of scarring tissue in the residual portion of the clivus, without evidence of lesions suggesting tumor residue or recurrence. The endoscopic 
findings were free of recurrent disease. The patient presented mild complications associated to radiotherapy: actinic fibrosis, grade II xerostomia without trismus and remained with vision loss in the right eye and hearing loss that she presented in the preoperatively. (Figure 4).

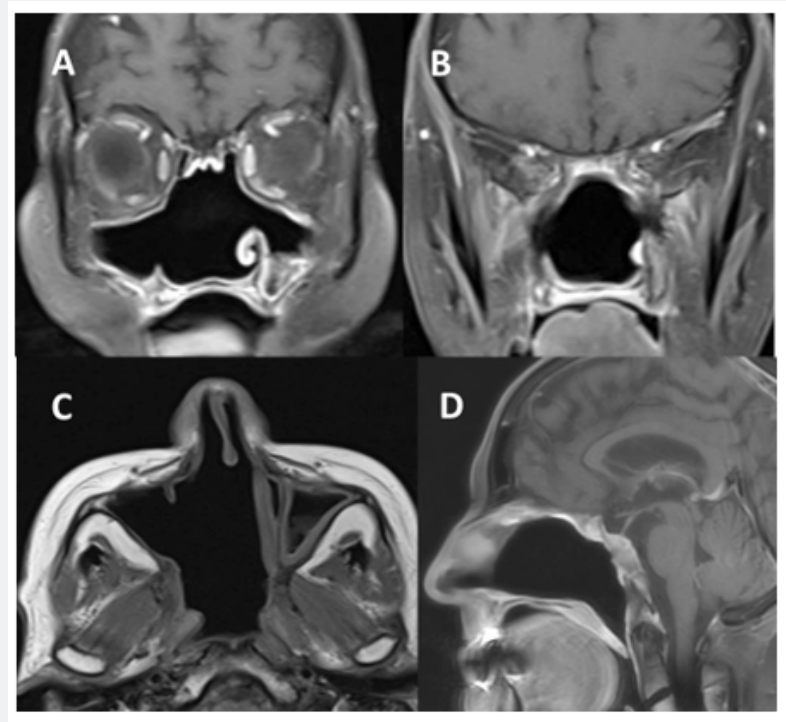

Figure 4: The latest follow-up images in January 2020 (35 months after treatment) showed postoperative changes in the endonasal approach with the presence of scarring tissue in the residual portion of the clivus, without evidence of lesions suggesting tumor residue or recurrence. A y B: coronal Plane, C: Axial plane y D: Sagital Plane.

\section{Discussion}

The ACC tumor of the paranasal sinuses and skull base is a tumor that presents an indolent clinical pattern with a tendency towards perineural invasion. It represents 3 to $5 \%$ of all head and neck cancers [9] and about 6 to $10 \%$ of malignant tumors of the salivary glands [9]. With a low incidence of lymph node and distant metastases, between 3 and 5.5\% [7]. Sayan M et al., Estimate that the annual global incidence rate is 0.4 to 13.5 cases per 100,000 habitants [8]. Advanced $\mathrm{T}$ stage reflects a poor prognosis for patients, and 10-year survival is very low $[3,6,9]$. It has been found that the histological subtype (tubular, cribriform, solid, or mixed) and the subsite of origin in the paranasal sinuses influences the prognosis, classifying the solid subtype as the most malignant and those originated in the ethmoid and sphenoid sinus as those with the lowest disease-specific survival in 5 years, reported in $25 \%$ $[4,6,14]$.

The presence of lymph nodes in the neck does not have a significant impact on survival, but it does increase the risk of developing distant metastases since this can occur even in the absence of locoregional recurrence [15]. Some recent studies have shown that the expression of c-Kit mutations is associated with a significantly worse prognosis, whereas the expression of EGFR provides better survival at 3 years [10]. Historically, the main treatment for ACC has been surgical resection of the primary tumor followed by adjuvant radiation therapy (RT) [6].
This has been established as an optimal therapy for this type of tumors $[5,11,12]$. However, in cases in which the tumor is locally advanced with proximity to vital neurovascular structures, treatment represents a real challenge for the treating team.

According to the study by Sayan et al., Adjuvant RT can improve local and regional disease control [8]. However, Ellington et al. reported that RT does not provide any benefit on overall survival (OS) [16]. Also, we found in a prospective french study adjuvant RT was indicated in $57 \%$ of cases for locoregional control, mainly in cases where the surgery is micro or macroscopically incomplete and in high-grade tumors ( $>30 \%$ solid component) making the adjuvant RT a good therapy for these patients [6]. Nevertheless, the benefit of adjuvant radiotherapy on overall survival (OS) has not been demonstrated with randomized studies, and therefore it is difficult to establish a treatment protocol that is widely accepted $[10,12]$. There is evidence of a slight increase in disease-specific survival at 5 years to $73.5 \%$ with adjuvant RT following surgical resection, taking into consideration that the newest radiation techniques as IMRT, allows reduction in the dose directed towards organs at risk, compared to conventional bilateral opposing field techniques [17].

In this case the patient received Radiotherapy technique IMRT, to provide doses from 2Gy to 66Gy in the surgical field, two months after the total resection of the tumor via trans nasal approach. Resulting in a low morbidity radiation technique with low risk for 
associated complications. Follow-up MRI and periodic endoscopic evaluations were performed for 2.5 years after definitive treatment, since it is the best modality to identify recurrent lesions, evaluate the skull base, perineural spread and intracranial extension [7]. During the follow-up period, no recurrent lesions were found. The outcomes of our case are like the findings of Sayan et al, Ali $S$ et al and Meyers et al, who reported that surgery followed by adjuvant RT can improve local and regional control of the disease $[8,17,18]$, considering this combination, the best approach to the treatment of non-metastatic ACC $[5,11,12]$. Further research with prospective randomized analytical studies will present type I and II evidence on the treatment of adenoid cystic carcinoma of the sinonasal and skull base region that is necessary to establish widely accepted treatment protocols $[10,12]$.

\section{Conclusion}

This clinical case illustrates the multidisciplinary approach that these patients with complex sinonasal malignancies require to achieve adequate control of their disease. Management by an extended endoscopic endonasal procedure with adjuvant radiation therapy (IMRT), appears to be an effective oncological alternative to well-selected cases of patients with locally advanced ACC tumors of the sinonasal tract.

\section{Acknowledgement}

Mario Melo MD, Pathologist, Instituto Nacional de Cancerología, Bogotá D.C. For providing the histopathology report and photos.

\section{References}

1. Lupinetti A, Roberts D, Williams M, Michael E Kupferman, David I Rosenthal, et al. (2007) Sinonasal adenoid cystic carcinoma: The M. D. Anderson Cancer Center experience. Cancer 110: 2726-2731.

2. Cao C, Ge M, Chen X, Xu J, Chen C (2017) Clinical outcomes and prognostic factors of salivary gland adenoid cystic carcinomas: a case control study. Oral Surg Oral Med Oral Pathol Oral Radiol 123: 531-535.

3. Andrade MF, de Faria PR, Cardoso SV, M R M Santos, F L Dias, et al. (2014) Adenoid cystic carcinoma of the maxillary sinus: A clinicalpathological report of 10 years of experience from a single institution. Int J Oral Maxillofac Surg 43: 1313-1318.

4. Amit M, Binenbaum Y, Sharma K, Naomi Ramer, Ilana Ramer, et al. (2013) Adenoid cystic carcinoma of the nasal cavity and paranasal sinuses: A meta-analysis. J Neurol Surgery, Part B Skull Base 74: 118125
5. Wiseman SM, Popat SR, Rigual NR, Wesley L Hicks Jr, James B Orner, et al. (2002) Adenoid cystic carcinoma of the paranasal sinuses or nasal cavity: A 40-year review of 35 cases. Ear Nose Throat J 81: 510-517.

6. Hallacq P, Labrousse F, Roullet B, Orsel S, Bessede JP, et al. (2001) Adenoid cystic carcinomas invading the skull base. Report of four cases and review of the literature. Neurochirurgie 47: 542-551.

7. Gendeh BS, Zahedi FD, Ahmad H, Kew TY (2013) Adenoid cystic carcinoma of the sinonasal tract: Outcome of endonasal endoscopic surgery at five-year follow up. J Laryngol Otol 127: 511-515.

8. Sayan M, Vempati P, Miles B, Marita Teng, Eric Genden, et al. (2016) Adjuvant therapy for salivary gland carcinomas. Anticancer Res 36: 4165-4170.

9. He S, Li P, Zhong Q, Lizhen Hou, Zhenkun Yu, et al. (2017) Clinicopathologic and prognostic factors in adenoid cystic carcinoma of head and neck minor salivary glands: A clinical analysis of 130 cases. Am J Otolaryngol 38: 157-162.

10. Bell D, Roberts D, Kies M, Rao P, Weber RS, et al. (2010) Cell typedependent biomarker expression in adenoid cystic carcinoma. Cancer 116: 5749-5756.

11. Pommier P, Liebsch NJ, Deschler DG, Derrick T Lin, James F McIntyre, et al. (2006) Proton beam radiation therapy for skull base adenoid cystic carcinoma. Arch Otolaryngol Head Neck Surg 132: 1242-1249.

12. Iseli TA, Karnell LH, Graham SM, G F Funk, J M Buatti, et al. (2009) Role of radiotherapy in adenoid cystic carcinoma of the head and neck. J Laryngol Otol 123: 1137-1144.

13. Volpi L, Bignami M, Lepera D, Apostolos Karligkiotis, Andrea Pistochini, et al. (2019) Endoscopic endonasal resection of adenoid cystic carcinoma of the sinonasal tract and skull base. Laryngoscope 129:1071-1077.

14. Khan AJ, DiGiovanna MP, Ross DA, Sasaki CT, Carter D, et al. (2001) Adenoid cystic carcinoma: A retrospective clinical review. I J Cancer 96: 149-158.

15. Lloyd S, Yu JB, Wilson LD, Decker RH (2011) Determinants and patterns of survival in adenoid cystic carcinoma of the head and neck, including an analysis of adjuvant radiation therapy. Am J Clin Oncol Cancer Clin Trials 34: 76-81.

16. Ellington CL, Goodman M, Kono SA, William Grist, Trad Wadsworth, et al. (2012) Adenoid cystic carcinoma of the head and neck: Incidence and survival trends based on 1973-2007 Surveillance, Epidemiology, and End Results data. Cancer 118: 4444-4451.

17. Ali S, Palmer F, Katabi N, Lee N, Shah J, et al. (2017) Long-term local control rates of patients with adenoid cystic carcinoma of the head and neck managed by surgery and postoperative radiation. Laryngoscope 127: 2265-2269.

18. Meyers M, Granger B, Herman P, F Janot, R Garrel, et al. (2016) Head and neck adenoid cystic carcinoma: A prospective multicenter REFCOR study of 95 cases. Eur Ann Otorhinolaryngol Head Neck Dis 133: 13-17. 
(C) This work is licensed under Creative DOI: 10.19080/GJO.2021.24.556126

\section{Your next submission with Juniper Publishers will reach you the below assets}

- Quality Editorial service

- Swift Peer Review

- Reprints availability

- E-prints Service

- Manuscript Podcast for convenient understanding

- Global attainment for your research

- Manuscript accessibility in different formats

( Pdf, E-pub, Full Text, Audio)

- Unceasing customer service

Track the below URL for one-step submission https://juniperpublishers.com/online-submission.php 